\title{
Self-Assembled Asymmetric Block Copolymer Membranes: Bridging the Gap from Ultra- to Nanofiltration
}

\author{
Haizhou Yu, ${ }^{[\mathrm{ac}]}$ Xiaoyan Qiu, ${ }^{[\mathrm{a}]}$ Nicolas Moreno, ${ }^{[\mathrm{b}],[\mathrm{cc}]}$ Zengwei Ma, ${ }^{[\mathrm{e]}}$ Victor Manuel Calo, ${ }^{[\mathrm{c}],[\mathrm{d}]}$ Suzana P. \\ Nunes $^{[b]}$ and Klaus-Viktor Peinemann ${ }^{\star[a]}$
}

Dedication ((optional))

\begin{abstract}
Self-assembly of block copolymers is an emerging strategy to produce isoporous ultrafiltration membranes. But till today it was not possible to bridge the gap from ultra- to nanofiltration and decrease the pore size of self-assembled block copolymer membranes to the sub $5 \mathrm{~nm}$ range without post-treatment. Here we report that blends of two chemically interacting copolymers can form highly porous membranes by self-assembly with pore diameters as small as $1.5 \mathrm{~nm}$. The membrane containing an ultra-porous $60-\mathrm{nm}$ thin separation layer can totally reject solutes with molecular weights of $600 \mathrm{~g} / \mathrm{mol}$ in aqueous solutions with a water flux more than an order of magnitude higher than permeances of commercial nanofiltration membranes. Simulation of the membrane formation process by dissipative particle dynamics (DPD) allowed us to explain the dramatic pore size reduction observed aligned to high water flux.
\end{abstract}

Nanoporous membranes are an important tool for water softening, the removal of micro-pollutants, demineralization of organic solutes, protein separation and the recovery of dyes from waste water. ${ }^{[1,2]}$ Although a wide variety of nanoporous membrane systems has been developed, a number of challenges in their production still exists. ${ }^{[3-6]}$ These include improving the permeability of the membranes with simultaneous and efficient rejection of specific molecules, up-scaling the production process, reducing membrane fouling and reproducing membrane performance. Self-assembly of well-defined block copolymers is a key strategy in the fabrication of functional nanostructured materials. ${ }^{[7,8]}$ Amphiphilic BCPs are able to selfassemble into well-defined nanoscale structures with a domain spacing that depends on the balance of repulsive interactions

[a] $\quad$ Dr. H. Z. Yu, ${ }^{+}$Dr. X. Y. Qiu, ${ }^{+}$Prof. K. V. Peinemann Advanced Membranes and Porous Materials Center 4700 King Abdullah University of Science and Technology (KAUST) Thuwal 23955-6900 (Kingdom of Saudi Arabia) Email: klausviktor.peinemann@kaust.edu.sa

$\left.{ }^{+}\right]$These authors contributed equally to this work

[b] Dr. N. Moreno, Prof. S. P. Nunes Biological and Environmental Science and Engineering Division (KAUST)

Thuwal 23955-6900 (Kingdom of Saudi Arabia)

[c] Dr. N. Moreno, Prof. V. M. Calo

Center for Numerical Porous Media (KAUST)

Thuwal 23955-6900 (Kingdom of Saudi Arabia)

[d] Prof. V. M. Calo

Earth Science and Engineering \& Applied Mathematics and Computational Science (KAUST)

Thuwal 23955-6900 (Kingdom of Saudi Arabia)

[e] Dr. Z.W. Ma

School of Optoelectronic Information, Chongqing University of

Technology

Chongqing 40054 (China)

Supporting information for this article is given via a link at the end of the document.((Please delete this text if not appropriate)) between dissimilar segments and the conformational entropy loss of the distinct blocks. ${ }^{[9-11]}$ Several years ago Peinemann et al. reported a simple and scalable technique for fabricating integral asymmetric isoporous block copolymer membranes. ${ }^{[12]}$ Since then our group studied the formation mechanism and tuned the pore size between 20 and $3 \mathrm{~nm} \cdot{ }^{[13-15]}$ The sub $10 \mathrm{~nm}$ pores were obtained by electroless gold deposition. ${ }^{[13]}$ However, according to Hagen-Poiseuille's equation: $d v / d t=\left(\pi R^{4} \Delta p\right) /(8 \eta L)$,

the flux $(d v / d t)$ decreases rapidly as the pore radius decreases. To address this decrease in flux, we focused on the fabrication of nanoporous membranes with high water permeability by combining the self-assembly of $A B / A C B C P$ blends with nonsolvent-induced phase separation. We investigated the blending of $\mathrm{BCPs}$ because of the simplicity of the process.
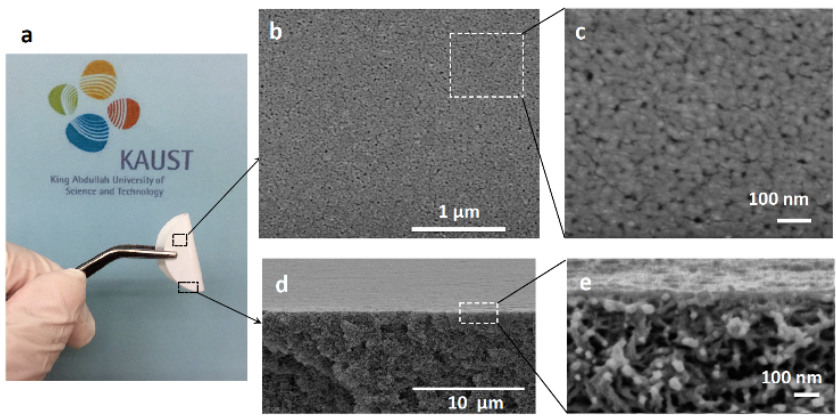

Figure 1. (a) Photograph showing the flexibility of the blended $B C P P_{144}-b$ $\mathrm{PAA}_{22} / \mathrm{PS}_{1807}-b-\mathrm{P}_{4} \mathrm{VP}_{609}$ membrane with a 6:1 molar ratio; (b, c) FESEM images of the membrane surface at different magnifications, showing a large number of small pores; (d, e) Cross-section FESEM images showing the thin top layer and the sponge-like bottom layer at different magnifications.

Bulk morphologies, thin films and micelles of BCP blends have been previously studied whereas only a few studies of membranes have been conducted. ${ }^{[16-20]}$ The most recent publication on blended BCP membranes describes the blends of two $A B$ block copolymers with different molecular weights and block lengths. ${ }^{[21]}$ In that study, the pore size could be tailored, but the pore diameter could not be decreased below the pore diameter of the single-component block copolymer membrane. Moreover, reported pore sizes in the previous studies on BCP membranes are consistently equal to or larger than $20 \mathrm{~nm}$. Here, we selected blends of interacting $A B / A C$ copolymers and decreased the pore size to the nanofiltration range (smaller than $3 \mathrm{~nm}$ ). Two di-BCPs, i.e., high-molecular-weight $\mathrm{PS}_{1807}-b-\mathrm{P}_{4} \mathrm{VP}_{609}$ and low-molecularweight $\mathrm{PS}_{144}-b-\mathrm{PAA}_{22}$, in which $\mathrm{P} 4 \mathrm{VP}$ and $\mathrm{PAA}$ can interact 
through hydrogen bonds (Supporting Information Scheme S1), were chosen. Instead of directly patterning the pores, we discovered that nanoscale voids, giving rise to the pores of the blended BCP membrane, are formed spontaneously between the densely packed spherical micelles.

The new blended BCP membranes were fabricated by introducing different amounts of $\mathrm{PS}_{144}-b-\mathrm{PAA}_{22}$ into $\mathrm{PS}_{1807}-b$ $\mathrm{P} 4 \mathrm{VP}_{609} / \mathrm{DMF} /$ dioxane/acetone mixtures. The initial PS- $b-\mathrm{P} 4 \mathrm{VP}$ weight was constant in all experiments, while the $\mathrm{PS}_{144}-b-\mathrm{PAA}_{22}-$ to-PS ${ }_{1807}-b-\mathrm{P}_{4} \mathrm{VP}_{609}$ molar ratio ranged from $2.5: 1$ to $9: 1$. A flexible membrane (Figure 1a) was obtained comprising a thin layer on top of a sponge-like layer. Figures $1 \mathrm{~b}$ and $1 \mathrm{c}$ show the blended BCP membrane with a $\mathrm{PS}_{144}-b-\mathrm{PAA}_{22}$-to-PS $1807-b-$ $\mathrm{P} \mathrm{VP}_{609}$ molar ratio of $6: 1$, suggesting a high pore density. The average thickness of the top layer as shown in the cross-section SEM images (Figures $1 \mathrm{~d}$ and 1e) was only ca. $60 \mathrm{~nm}$. To evaluate the pore size of the blended BCP membranes, we filtered protoporphyrin-IX $\left(M_{\mathrm{w}}=562.6 \mathrm{~g} / \mathrm{mol}, 0.01 \mathrm{mg} / \mathrm{ml}\right)$ and lysine $\left(M_{\mathrm{w}}=146.2 \mathrm{~g} / \mathrm{mol}, 1 \mathrm{mg} / \mathrm{ml}\right)$ solutions through the membranes. The membranes were tested in an Amicon stirred cell at a pressure of 1.37 bar. We manufactured membrane areas of $50 \mathrm{~cm}^{2}$ or larger; the final membrane discs for testing had diameters of $2.2 \mathrm{~cm}$.
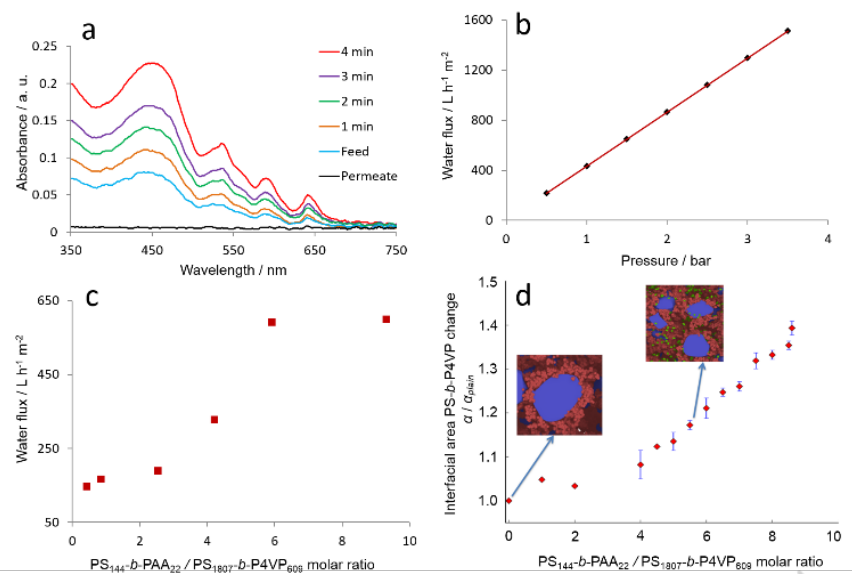

Figure 2. (a) Ultraviolet-visible absorption shows the change in concentration of the aqueous solution of protoporphyrin-IX with filtration time and indicates the complete rejection of protoporphyrin-IX by the blended BCP membrane with a 6:1 $\mathrm{PS}_{144}-b-\mathrm{PAA}_{22}$-to-PS ${ }_{1807}-b-\mathrm{P}_{4} \mathrm{VP}_{609}$ molar ratio; (b) Pressuredependent changes in the permeability of pure water through the blended BCP membrane in (a); (c) The curve shows how the water permeability changes with the content of $\mathrm{PS}_{144}-b-\mathrm{PAA}_{22}$ in the blended BCP membrane; (d) Change in interfacial area $(\alpha)$ between PS and P4VP domains with the content of $\mathrm{PS}_{144}-b-\mathrm{PAA}_{22}$, relative to the plain system $\left(\alpha_{\text {plain }}\right)$

Figure $2 \mathrm{a}$ shows that the retentate concentration of protoporphyrin-IX increased with filtration time, and no protoporphyrin-IX could be detected in the permeate. This means that water passed through the membrane, while protoporphyrin-IX was rejected by the blended BCP membrane. However, lysine freely passed through the membrane without rejection. Protoporphyrin-IX and lysine have diameters of 1.47 $\mathrm{nm}$ and $0.9 \mathrm{~nm}$ (Supporting Information Figure S1), respectively, as characterized by $\mathrm{DLS}^{[2]}$ indicating that the effective pore diameter of the blended BCP membrane was smaller than ca. $1.5 \mathrm{~nm}$. Additionally, Brunauer-Emmett-Teller (BET) analysis, calculated from the $\mathrm{N}_{2}$ adsorption isotherm using the BarrettJoyner-Halenda (BJH) method, shows a sharp peak for the pore width at around $1.7 \mathrm{~nm}$ (Supporting Information Figure S5) and a broader distribution above $10 \mathrm{~nm}$. The peaks for membranes were observed to be asymmetric and the peak in the lower size range is consistent with the sharp cut-off of the membrane's selective layer, while the broader distribution in the larger size range corresponds to sub-layers with increasing pore structures formed by macrophase separation. More detailed filtrations of different solutes through the membranes are presented in Supporting Information Table S1.

Another remarkable property of this membrane is that the fluxes were as high as $540 \mathrm{~L} \mathrm{~m}^{-2} \mathrm{~h}^{-1}$ for the protoporphyrin-IX solution and $592 \mathrm{~L} \mathrm{~m}^{-2} \mathrm{~h}^{-1}$ for pure water at a pressure of 1.37 bar. Compared with commercial membranes with similar pore sizes, our blended BCP membranes are manufactured using a simple one-step fabrication process; they have a narrow pore size distribution, high water permeability and high scale-up potential. In addition, the water flux through the membrane with the 6:1 $\mathrm{PS}_{144}-b$-PAA 22 -to-PS ${ }_{1807}-b-\mathrm{P}_{4} \mathrm{VP}_{609}$ molar ratio increased linearly with pressure in the range of 0.5-3.5 bar as shown in Figure $2 b$, indicating the stability of the membrane.

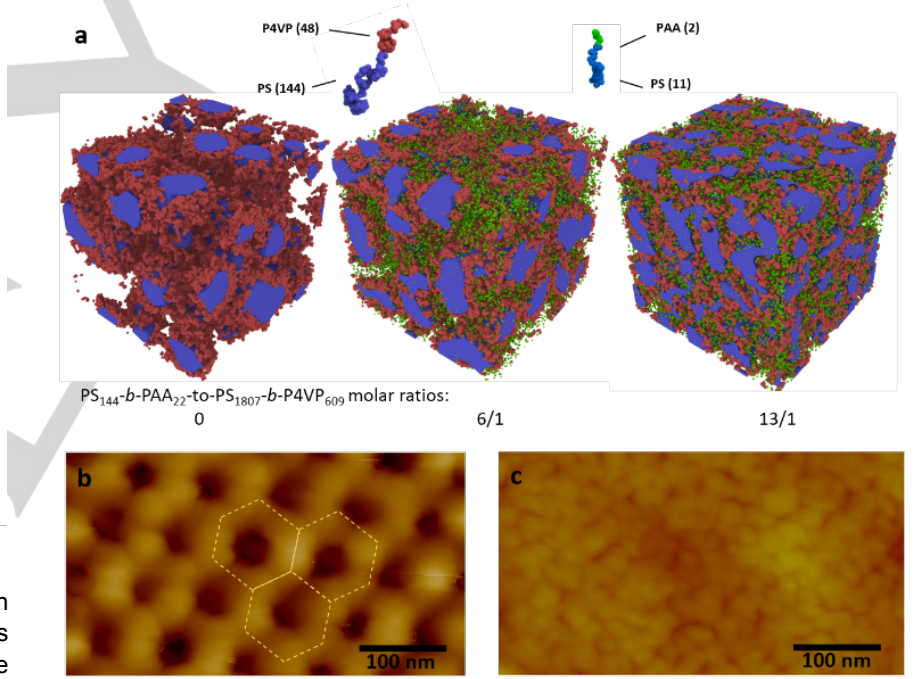

Figure 3. (a) Self-assembled structures obtained by computational simulations (DPD), under experimental conditions (17\% of PS- $b$-P4VP) with different $\mathrm{PS}_{144}-b-\mathrm{PAA}_{22}$-to-PS $1807-b-\mathrm{P}_{4} \mathrm{VP}_{609}$ molar ratios. Solvent particles and $\mathrm{PS}$ beads of $\mathrm{PS}_{144}-b-\mathrm{PAA}_{22}$ are omitted to facilitate the visualization. (b, c) AFM images show the surfaces of (b) the pure $\mathrm{PS}_{1807}-b-\mathrm{P}_{4} \mathrm{VP}_{609}$ and (c) blended BCP membrane with a $6: 1 \mathrm{PS}_{144}-b-\mathrm{PAA}_{22}$-to-PS ${ }_{1807}-b-\mathrm{P}_{4} \mathrm{VP}_{609}$ molar ratio. The figures indicate the morphological transition on the surface from quasihexagonal (dashed circles) to densely packed spherical micelles leading to smaller pores and higher pore density.

The formation of nanopores with ultrafast water permeation is guided by the self-assembly of the two copolymer molecules in the solution. A special feature of the plain PS- $b$-P4VP membrane formation in a selective solvent mixture is the micelle formation and its supramolecular assembly in hexagonal order. This order is practically immobilized by immersion in water, a 
non-solvent for the block copolymer. At the same time, phase separation by spinodal decomposition takes place, leading to the consolidation of a more disordered, asymmetric porous substructure. ${ }^{[14,15]}$ The ordered hexagonal top layer of the plain PS- $b$-P4VP can be seen in Figure $3 \mathrm{~b}$, imaged by an atomic force microscope (AFM). We previously investigated the mechanism of pore formation in membranes obtained from single PS-b-P4VP copolymers by small-angle X-ray scattering $(\mathrm{SAXS})^{19}$, cryo microscopy ${ }^{[22,23]}$ and in-situ grazing-incidence $\mathrm{SAXS}^{[23]}$ and demonstrated how self-assembly in solution is relevant to the process and how it is affected by the copolymer concentration and solvent composition. With the introduction of PS- $b$-PAA to the PS- $b$-P4VP solution, the micellar order is disturbed and a much denser surface is obtained, as shown in Figure $3 \mathrm{c}(\mathrm{AFM})$, as well as $1 \mathrm{~b}$ (field emission scanning electron microscopy, FESEM). The assembly in solution as well as the final membrane morphology is more complex. Vyhnalkova et al. ${ }^{[24,25]}$ recently reported a detailed investigation of the morphology of PS- $b$-P4VP/PS- $b$-PAA mixtures in relatively diluted solutions containing water. They emphasized the complexity and the interplay between the copolymer lengths as well as the ionic character, solubility and response to protonation of these mixtures. The solutions we used for membrane casting
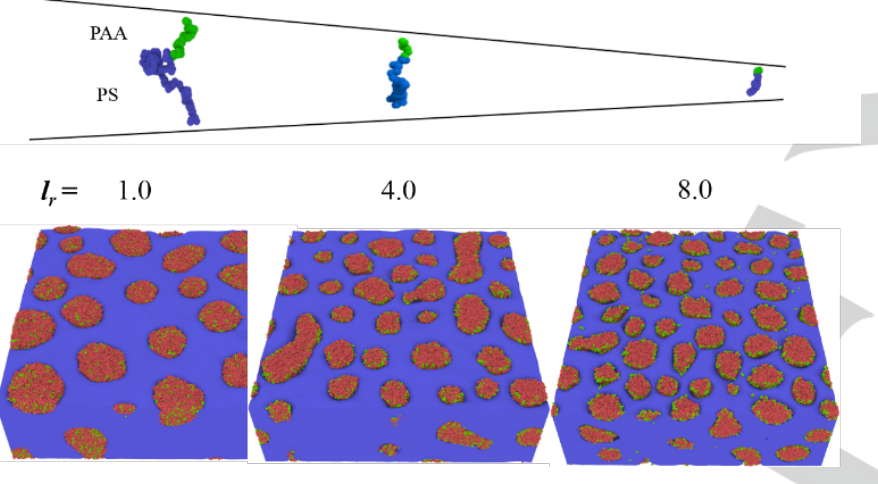

Figure 4. DPD results showing the effect of the length of the PS- $b$-PAA copolymer on the self-assembly morphology in solution. $I_{r}$ is the length ratio, where $I_{r}=N_{P S-b-P 4 V P} / N_{P S-b-P A A}$. The $\mathrm{PS}_{1807}-b-\mathrm{P}_{4} \mathrm{VP}_{609}$ concentration remained constant at 25 wt $\%$, with a molar ratio $n_{\text {PS-b-PAA }} / n_{\text {PS-b-P4VP }}$ of 8.6:1. PS blocks are depicted in blue, while the red and green domains represent P4VP and PAA segments, respectively. Solvent particles are omitted to facilitate the visualization. As $I_{r}$ increase the PS- $b$-PAA is preferentially localized at the interface.

were in a much higher concentration range than those used by Vyhnalkova et al.

To understand the assembly changes in the presence of PS- $b$-PAA in the concentration range relevant to membranes, we modeled the block copolymer blends using dissipative particle dynamics (DPD), a method introduced by Hoogerbrugge and Koelman. ${ }^{[26]}$ We previously used DPD simulation to explain the formation of plain PS- $b$-P4VP membranes. ${ }^{[22]}$ While PS $_{1807-}$ $b$-P4VP 609 formed micelles in the solvent mixture investigated (DMF/dioxane/acetone), the segregation was not strong in the case of pure $\mathrm{PS}_{144}-b-\mathrm{PAA}_{22}$ due to the relatively low $A A$ block size. Segregation is favored when the thermodynamic interactions multiplied by blocks sizes are high (Supporting
Information Figure S2, Tables S3-S5). When the two copolymers are mixed, complexes are formed due to hydrogen-bonding interactions, ${ }^{[27]}$ and the PAA blocks can penetrate the shell of the PS- $b$-P4VP micelles, leading to the morphology changes depicted in Figure 3a and in Supporting Information Figure S3. The DPD simulation shows that the radius of gyration for PS- $b$ P4VP chains decreased as the PS- $b$-PAA ratio increased, as did the characteristic segregation domain size in the final structured membrane (Figure 3a). Correspondingly, the AFM results indicate the decrease of micellar size from ca. $35 \mathrm{~nm}$ for the pure PS-b-P4VP membrane (Figure $3 b$ ) to $c a .15 \mathrm{~nm}$ or less for the blended BCP membrane (Figure 3c). Smaller pores are formed. Conversely, because of the hydrophobic character (PS blocks) of PS-b-PAA, we believe that they promote the morphological transition by inserting their hydrophobic chains into PS- $b$-P4VP micellar aggregates (Supporting Information Figure S3), also leading to an increase in the entropic constraint. ${ }^{[28]} \mathrm{A}$ morphological transition from quasi-hexagonal to densely packed spherical micelles also helps to relieve this entropy strain (Figure 3), leading to smaller, highly dense pores. The final surface morphology is the result of cooperation between these two factors, i.e., hydrogen bonding between P4VP and PAA units and the hydrophobic tails of PS- $b$-PAA inserting into the PS- $b$-P4VP micellar cores. A clear change in the water flux as the relative amount of PS- $b$-PAA increases is shown in Figure $2 c$. The block length of PS- $b$-PAA also plays a critical role in the fabrication of the blend membrane. Aggregation is likely to happen in blends of $\mathrm{PS}_{1807}-b-\mathrm{P}_{4} \mathrm{VP}_{609}$ and large-molecular-weight $\mathrm{PS}_{673}-b-\mathrm{PAA}_{180}$ as confirmed both by experimental (not shown here) and simulation results (Figure 4, Supporting Information Figures S3 and S4). The effect of the length-ratio $\left(l_{r}=N_{\text {PS-b-P4VP }} / N_{\text {PS-b-PAA }}\right)$ on the overall self-assembly is evident in the DPD results depicted in Figure 4. Lower values of $I_{r}$ correspond to longer PAA chains and lead to strong associations with P4VP domains and an overall increase in the domain size. Figure $4 \mathrm{~b}$ simulates the morphology after the concentration of polymer increases by solvent evaporation and addition of water (a poor solvent in this case) to the system, demonstrating the formation of a large number of smaller domains in the presence of small PS- $b$-PAA copolymers. These domains give rise to the membrane's pores.

The faster water transport through the blended BCP membrane (Figure 2c and Supporting Information Table S1) is a consequence of the exceptional pore density, the thin top layer, and the highly hydrophilic PAA blocks. The number of pores increases dramatically after the addition of PS- $b$-PAA (Figures $3 b$ and $3 c$ ), which is partially located in the pore walls. A clear transition in the permeability characteristics is seen for $\mathrm{PS}_{144}-b$ $\mathrm{PAA}_{22}$-to-PS ${ }_{1807}-b-\mathrm{P}_{4} \mathrm{VP}_{609}$ molar ratios between $4: 1$ and $6: 1$. The increase in hydrophilicity is confirmed by the contact angle values and the water-membrane adhesion (Supporting Information Table S2). The transition in permeability can be also associated with the reduction in the micellar size. This trend is consistent with the increment in interfacial area $(\alpha)$ between PS and P4VP domains measured in our simulations (Figure 2c). The abrupt change in area at molar ratios around $4: 1$, reflects the reduction in the characteristic domain-size.

The advantage of this technique is that the nanopore membrane fabrication is simple compared with post-deposition 
fabrication processes ${ }^{[13,29,30]}$ and synthesis of new polymers. ${ }^{[31]}$ Our simulations suggest that a variety of copolymers can be used with this technique. It should also be possible to scale-up the blended BCP membrane fabrication process by combining high separation factors with high fluxes.

\section{Experimental Section}

Membrane preparation. Polystyrene- $b$-poly(4-vinylpyridine) block copolymer $\left(\mathrm{PS}_{1807}-b-\mathrm{P}_{4} \mathrm{VP}_{609}, M_{n}=252,000 \mathrm{~g} \mathrm{~mol}^{-1}\right)$ and Polystyrene- $b$ poly(acrylic acid) block copolymers ( $P_{144}-b-P_{A} A_{22}, M_{n}=16,600 \mathrm{~g} \mathrm{~mol}^{-1}$ and $\mathrm{PS}_{673}-b-\mathrm{PAA}_{180}, M_{n}=83,000 \mathrm{~g} \mathrm{~mol}^{-1}$ ) were purchased from Polymer Source, Inc., Canada. The subscripts indicate the number of repeat units of the blocks. All other chemicals were supplied by Aldrich. The blended $\mathrm{BCP}$ membranes were cast from a polymer solution mixture of $\mathrm{PS}_{144}-b$ $\mathrm{PAA}_{22}$ and $\mathrm{PS}_{1807}-b-\mathrm{P}_{4} \mathrm{VP}_{609} / \mathrm{DMF} /$ dioxane/acetone on a Hirose $\mathrm{TH} 100$ polyester support, using casting blades with $200 \mu \mathrm{m}$ gaps. The $\mathrm{PS}_{1807}-b$ $\mathrm{P}_{4} \mathrm{VP}_{609}$ weight and the organic solvent composition remained constant, i.e., 18 wt \% copolymer, 24 wt \% N, N-dimethyl formamide (DMF), 42 wt $\%$ dioxane, and 16 wt \% acetone for both the blended BCP membrane and the plain PS- $b-\mathrm{P} 4 \mathrm{VP}$ membrane, while the molar ratio $\left(n_{\mathrm{PS}-b-\mathrm{PAA}} / n_{\mathrm{PS}-b-}\right.$ $\mathrm{P} 4 \mathrm{VP}$ ) of $\mathrm{PS}_{144}-b-\mathrm{PAA}_{22}$ to $\mathrm{PS}_{1807}-b-\mathrm{P}_{4} \mathrm{VP}_{609}$ ranged from $2.5: 1$ to $9: 1$ in the blended BCP membrane. The solvent was allowed to evaporate for up to $10 \mathrm{~s}$ at room temperature, and the film was then immersed in a non-solvent bath (Milli-Q water, 18.2 $\mathrm{M} \Omega$ ) at room temperature.

Field emission scanning electron microscopy (FESEM) images were obtained using a FEI Quanta 600 series microscope at $5 \mathrm{kV}$ with a working distance of $10 \mathrm{~mm}$. Atomic force microscopy (AFM) images were obtained on an ICON Veeco microscope operating in the tapping mode using commercial silicon TM AFM tips (model MPP 12100). Additional details for the characterization of the membranes including contact angle, $B E T$, and DLS analyses are described in the Supporting Information. The water flux measurements as well as the structure and sizes of the molecules for the rejection experiment are also explained in the Supporting Information. The rejection of the dyes, proteins and gold particles was monitored by a NanoDrop 2000/2000c spectrophotometer (Thermo Fisher Scientific).

Dissipative Particle Dynamics (DPD) simulation. The DPD simulations were conducted in the Large-scale Atomic/Molecula Massively Parallel Simulator (LAMMPS). ${ }^{[32]}$ We used the standard DPD units of length, $r_{c}=1$, mass $m=1$, energy $\epsilon=k_{B} T=1$, and time $t=1$. The particle density in our system was $\rho_{n}=3$ particles $/ r_{c}^{3}$. A time step of 0.04 T was used to restrict temperature fluctuations to be smaller than $1 \%$. The simulated systems were evolved for $1 e 10^{6}$ time steps. The analysis and visualization of the computational results were performed with in house software and OVITO. ${ }^{[3,34]}$ We used periodic boundary conditions in cubic simulation boxes of size $L_{b o x}=131 r_{c}$. The box size was defined to be proportional to the overestimated radius of gyration as $L_{b o x}=$ $z r_{o} N_{\text {PS-b-P4VP, }}^{u}$ where $z=6$ is the proportionality constant, $r_{o}=0.8 r_{c}$ is the equilibrium bond length between connected particles, $N_{P S-b-P 4 V P}=192$, and $u=0.63$. The value of $u$ is an overestimated parameter, since we are defining the box size as if the polymer coils were extended. Detailed parameters and descriptions are provided in the Supporting Information.

\section{Acknowledgements}

The authors gratefully acknowledge financial support from King Abdullah University of Science and Technology (KAUST) with special thanks for partial funding from the KAUST CRG2 project
"Charge-mosaic and Block Copolymer Membranes". They also thank Dr. Omar El Tall from the Analytical Core Lab at KAUST for help with the BET analysis. N.M and V.C. acknowledge the support of the Texas Advanced Computing Center TACC at the University of Texas at Austin and KAUST research computing center for providing HPC resources to perform the simulations and their excellent technical support. Zengwei $\mathrm{Ma}$ also acknowledges the National Natural Science Foundation of China (No. 21304110).

Keywords: nanofiltration $\cdot$ membrane $\cdot$ block copolymer $\bullet$ selfassembly

[1] L. A. Baker, S. P. Bird, Nature Nanotech. 2008, 3, 73-74.

[2] X. Peng, J. Jin, Y. Nakamura, T. Ohno, I. Ichinose, Nature Nanotech. 2009, 4, 353-357.

[3] C. C. Striemer, T. R. Gaborski, J. McGrath, P. M. Fauchet, Nature 2007, 445, 749-753.

[4] S. Karan, S. Samitse, X. Peng, K. Kurashima, I. Ichiinose, Science 2012, 335, 444-447.

[5] E. Krieg, H. Weissman, E. Shirman, E. Shimoni, B. Rybtchinski, Nature Nanotech. 2011, 6, 141-146.

[6] W. Lee, R. Ji, U. Gösele, K. Nielsch, Nature Mater. 2006, 5, 741-747.

[7] G. M. Whitesides, B. Grzybowski, Science 2002, 295, 2418-2421.

[8] A. Ruzette, L. Leibler, Nature Mater. 2005, 4, 19-31.

[9] F. S. Bates, G. H. Fredrickson, Phys. Today 1999, 52, 32-38

[10] E. A. Jackson, M. A. Hillmyer, ACS Nano 2010, 4, 3548-3553.

[11] J. Bang, U. Jeong, D. Y. Ryu, T. P. Russell, C. J. Hawker, Adv. Mater. 2009, 21, 4769-4792.

[12] K.-V. Peinemann, V. Abetz, P. F. W. Simon, Nature Mater. 2007, 6, 992996.

[13] H. Yu, X. Qiu, S. P. Nunes, K.-V. Peinemann, Angew. Chem. Int. Ed. 2014, 53, 1-6

[14] S. P. Nunes, R. Sougrat, B. Hooghan, D. H. Anjum, A. R. Behzad, L. Zhao, N. Pradeep, I. Pinnau, U. Vainio, K.-V. Peinemann, Macromolecules 2010, 43, 8079-8085.

[15] S. P. Nunes, M. Karunakaran, N. Pradeep, A. R. Behzad, B. Hooghan, R. Sougrat, H. He, K.-V. Peinemann, Langmuir 2011, 27, 10184-10190.

[16] W. Park, Y. Kim, J. Jeong, K. Kim, J.-K. Yoo, Y. Hur, J. Kim, E. L. Thomas, A. Alexander-Katz, Y. S. Jung, Sci. Rep. 2013, 3, 3190.

[17] M. Stoykovich, M. Müller, S. O. Kim, H. H. Solak, E. W. Edwards, J. J. de Pablo, P. F. Nealey, Science 2005, 308, 1442-1446.

[18] C. Tang, E. M. Lennon, G. H. Fredrickson, E. J. Kramer, C. J. Hawker, Science 2008, 322, 429-432.

[19] D. A. Christian, W. Tian, W. G. Ellenbroek, I. Levental, K. Rajagopal, P. A. Janmey, A. J. Liu, T. Baumgart, D. E. Discher, Nature Mater. 2009, 8, 843-849.

[20] J. Zhu, S. Zhang, K. Zhang, X. Wang, J. W. Mays, K. L. Wooley, D. J. Pochan, Nat. Commun. 2013, 4, 2297.

[21] M. Radjabian, V. Abetz, Adv. Mater. 2015, 27, 352-355.

[22] D. S. Marques, U. Vainio, N. M. Chaparro, V. M. Calo, A. R. Bezahd, J. W. Pitera, K.-V. Peinemann, S. P. Nunes, Soft Matter 2013, 9, 55575564.

[23] D. S. Marques, R. M. Dorin, U. Wiesner, D.-M. Smilgies, A. R. Behzad, U. Vainio, K.-V. Peinemann, S. P. Nunes, Polymer 2014, 55, 1327-1332.

[24] R. Vyhnalkova, A. H. Müller, A. Eisenberg, Langmuir 2014, 30, 1315213163.

[25] R. Vyhnalkova, A. H. Müller, A. Eisenberg, Langmuir 2014, 30, 50315040.

[26] P. J. Hoogerbrugge, J. M. V. A. Koelman, Europhys. Lett. 1992, 19, 155-160. 
[27] W. Zhang, L. Shi, Y. An, K. Wu, L. Gao, Z. Liu, R. Ma, Q. Meng, C. Zhao, B. He, Macromolecules 2004, 37, 2924-2929.

[28] N. S. Cameron, M. K. Corbierre, A. Eisenberg, Can. J. Chem. 1999, 77 1311-1326.

[29] P. Chen, T. Mitsui, D. Farmer, J. Golovchenko, R. G. Gordon, D. Branton, Nano Lett. 2004, 4, 1333-1337.

[30] D. Losic, G. Triani, P. J. Evans, A. Atanacio, J. G. Mitchell, N. H Voelcker, J. Mater. Chem. 2006, 16, 4029-4034.

[31] M. Barsbay, O. Güven, H. Bessbousse, T. L. Wade, F. Beuneu, M.-C. Clochard, J. Membr. Sci. 2013, 445, 135-145.

[32] S. Plimpton, Journal of Computational Physics 1995, 117, 1-19.
[33] A. Stukowski, Modelling and Simulation in Materials Science and Engineering 2010, 18, 015012.

[34] A. Stukowski, JOM 2013, 66, 399-407. 
Entry for the Table of Contents (Please choose one layout)

\section{Layout 1:}

\section{COMMUNICATION}

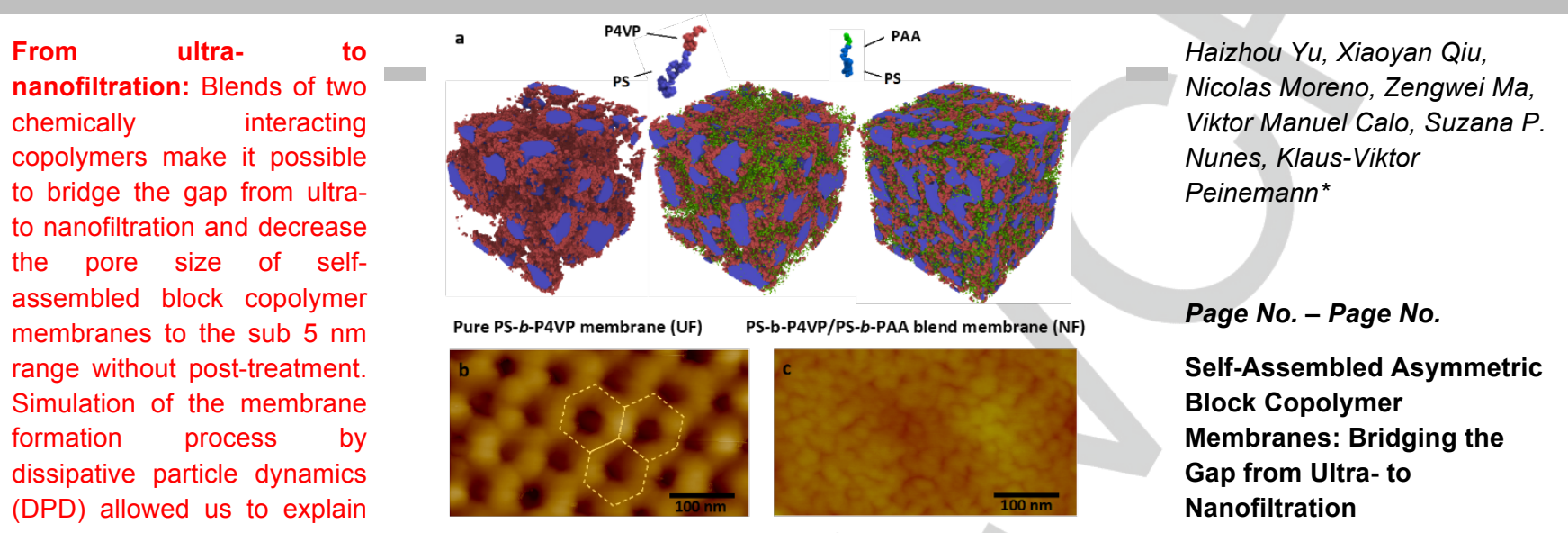

the dramatic pore size reduction observed aligned to high water flux. 